\title{
Response of different wheat varieties towards Azospirillum and phosphate solubilizing bacteria (PSB) seed inoculation
}

\author{
Animesh Pathak ${ }^{1 *}$, S. K. Chakrabarti ${ }^{2}$, Rajib Das ${ }^{3}$ and M K. Mandal ${ }^{3}$ \\ ${ }^{1}$ Department of Seed Science and Technology, M. S. Swaminathan School of Agriculture, Centurion University of \\ Technology and Management, Paralakhemundi, Dist. Gajapati-761211 (Odisha), INDIA \\ ${ }^{2}$ Department of Seed Science and Technology, Bidhan Chandra Krishi Viswavidyalaya, Mohanpur, Nadia-741252 \\ ( W.B.), INDIA \\ ${ }^{3}$ Department of Agronomy, Bidhan Chandra Krishi Viswavidyalaya, Mohanpur, Nadia-741252 (W.B.), INDIA \\ "Corresponding author. E-mail: animeshpathak88@gmail.com
}

Received: May 29, 2015; Revised received: August 27, 2015; Accepted: February 15, 2016

\begin{abstract}
Present investigation was undertaken to utilize Azospirillum and Phosphate Solubilizing Bacteria (PSB) to avail atmospheric nitrogen and soluble phosphate in soil for the wheat crop. The sonalika variety $\left(\mathrm{V}_{1}\right)$ responded best towards seed inoculation by Azospirillum and Phosphate Solubilizing Bacteria (PSB) for the character speed of germination which is regarded as very important indicator of seed vigour. Germination percentage for untreated control was $86.6 \%$ after one month of storage and found to be reduced to $76.67 \%$ after three month of storage but for treated Azospirillum in case of sonalika it was retained at $83.33 \%$. $V_{1} T_{1}, V_{2} T_{1}$ and $V_{3} T_{1}$ recorded improved germination even after six month of storage. The seedling characters like shoot and root length was greatly improved with $T_{1}$ and $T_{2}$ for almost the varieties included in this programme. The character vigour index greatly enhanced in case of $V_{1} T_{1}, V_{1} T_{2}, V_{3} T_{1}$ and $V_{3} T_{2}$ after one month and six months of storage. This work reported utilization of Azospirillum and PSB as seed inoculation for wheat varieties to enhance seed storability and seedling growth parameter. It can be concluded that, Azospirillum and PSB seed inoculation can be recommended for wheat for better seedling growth storability of seed
\end{abstract}

Keywords: Azospirillum, PSB, Seed inoculation, Varieties, Wheat

\section{INTRODUCTION}

Wheat (Triticum aestivum L.) can be classified as winter or spring growth habit based on flowering responses to cold temperatures. Biofertilizer like Rhizobium, Azotobacter, Azospirillum and blue green algae have been used for many years. Azospirillum inoculants are recommended mainly for wheat, sorghum, millets, maize, sugarcane and vegetable crops. Biofertilizer is defined as a substance which contains living organisms that when applied to seed, plant surface, or soil, colonize the rhizosphere or the interior of plant and promote growth by increasing supply or availability of primary nutrients to the host plant (Vessey, 2003). Biofertilizers are well recognized as an important component of integrated plant nutrient management for sustainable agriculture and hold a great promise to improve crop yield (Narula et al., 2005). Plant growth promoting rhizobacteria are free living microorganisms having beneficial effects on plants by colonizing their roots. They include such effects as the production of phytohormones; auxin, cytokinins and gibberellins (Garcia et al., 2001), enhancing release of the nutrients (Nautiyal et al., 2000). Azotobacter and Azospirillum have previously significantly increased wheat and barley yield in irrigated as well as in rainfed crops (Pauw De et al., 2008). To maintain the production potential as well as seed or grain quality of wheat, a concrete production technique is essential. One of the major essential elements for growth of plants is nitrogen. The Green Revolution (GR) technology adoption between 1960 to 2000 has increased wide varieties of agricultural crop yield per hectare which increased 12$13 \%$ food supply in developing countries. Nitrogen is required in large quantities for plants to grow, since it is the basic constituents of proteins and nucleic acids). Bio-fertilizers are the formulation of living organisms, which are able to fix atmospheric Nitrogen in the available from plants either by living frequency in soil or being associated symbiotically with plants (Subba Rao et al., 1993). Azospirilum, a bacterial fertilizer is highly beneficial micro-organism for cereals, cotton, plantation crops and other crops. The main function of Azospirillum inoculation is to assimilate atmospheric nitrogen and fix in soil and finally providing the growing plant. The aim of present investigation was to study the effect of Azospirillum and PSB inoculation on different seed quality parameters like speed of germination, storability and different seedling parameters like fresh and dry weight, seedling length and vigour index. 


\section{MATERIALS AND METHODS}

An experiment was carried out during the year of 2012 -2013 to 2013-2014 in the department of Seed Science and Technology, Faculty of Agriculture, Bidhan Chandra Krishi Viswavidyalaya, Mohanpur, Nadia, West Bengal. The experiment was done with three treatments and three replications with four varieties. The varieties were: $\mathrm{V}_{1}$ (Sonalika), $\mathrm{V}_{2}$ (PBW 443), $\mathrm{V}_{3}$ (HD 2821) and $\mathrm{V}_{4}$ (K 9107) and the treatments were$\mathrm{T}_{1}$ (Azospirillum), $\mathrm{T}_{2}$ (PSB) and $\mathrm{T}_{3}$ (Control) with thrice replicates. The data so obtained as described earlier were subjected to statistical analysis by Analysis of Variance method (Gomez and Gomez, 1984). The Standard Error mean $(\mathrm{SEm} \pm)$ and the value of critical difference $(\mathrm{CD})$ were computed to compare the difference between means have been provided in the tables of results. The data on germination and field emergence were transformed into angular (arcsine) values (Snedecor and Cochran, 1967) transformed values are given in the parenthesis and then subject to statistical analysis.

The statistical calculations were prepared by Factorial Design for analysis of data linked to varietal consequence and Completely Randomized Design (CRD) for laboratory data. All statistical analyses were done using SPSS (version 10.0, 1990) on a desktop Computer. The initial seed moisture content was $12 \%$. The seeds were stored in paper packet for one, three and six months of storage. The following seed quality parameters are recorded in the lab like Germination Percentage, Speed of germination, Root length, Shoot length, Seedling Fresh Weight, Dry Weight and Vigour index. The analysis of variance method (Cochran and Cox, 1963) was followed to analyze various data statistically. The significance of different sources of variation was tested by "Error Mean Square Method" of Fisher Snedecor's 'F' test at probability level 0.05 . Standard error of Mean (SEm \pm ) and critical difference (C.D.) have been estimated to compare the differences between means.

\section{RESULTS AND DISCUSSION}

The influence of different seed treatment on different varieties of wheat, particularly on the speed of germination which is regarded as very important indicator of seed vigour, found that the variety sonalika $\left(\mathrm{V}_{1}\right)$ was the best towards Azospirillum treatment (T1) and in treatment in PSB $\left(\mathrm{T}_{2}\right)$, V1 was best (Table 1). Speed of germination after one month of storage recorded highest volume in $\mathrm{V}_{1} \mathrm{~T}_{1}$ and $\mathrm{V}_{1} \mathrm{~T}_{2}$ than control. After three months of storage $V_{1} T_{1}$ and $V_{3} T_{2}$ responded better than control (Table 1). Response of different varieties after six months of storage was greatly pronounced in $\mathrm{V}_{1} \mathrm{~T}_{1}, \mathrm{~V}_{1} \mathrm{~T}_{2}, \mathrm{~V}_{2} \mathrm{~T}_{2}$ and $\mathrm{V}_{3} \mathrm{~T}_{2}$ for the character speed of germination (Table 1).

Germination percentage for control, after one month of storage was $86.67 \%$ which was found to be reduced to
$76.67 \%$, after three months of storage in case of $\mathrm{V}_{1}$ but for $\mathrm{T}_{1}$ it was retained at $83.33 \%$ after three months of storage (Table 2). $\mathrm{V}_{1} \mathrm{~T}_{1}, \mathrm{~V}_{2} \mathrm{~T}_{1}$ and $\mathrm{V}_{3} \mathrm{~T}_{1}$ recorded improved germination percentage than control. After six months of storage, control $\left(\mathrm{T}_{3}\right)$ exhibited loss of germination percentage to the extent of $63.33 \%$ in case of $V_{3}$ whereas the $\mathrm{V}_{3} \mathrm{~T}_{1}$ and $\mathrm{V}_{3} \mathrm{~T}_{2}$ maintained at $73.33 \%$ (Table 2). In case of soybean after 30 days plant maximum seed germination observed by biofertilizer treated plant $(82.85 \%)$ and minimum by chemical fertilizer treated plants $(0 \%)$ stated by Javed and Panwar (2013). Similar results were also reported by Strelec et al. (2010) to indicate significant influence of storage conditions on moisture content, germination and vigour changes during storage of wheat seeds, as well as varietal dependence of seed viability recorded. Response of different wheat varieties towards the character shoot length recorded high value for $V_{1} T_{1}, V_{1} T_{2}$ than control (Table 3 ). Similarly response was also obtained for $\mathrm{V}_{2} \mathrm{~T}_{1}, \mathrm{~V}_{2} \mathrm{~T}_{2}, \mathrm{~V}_{3} \mathrm{~T}_{1}$ and $\mathrm{V}_{3} \mathrm{~T}_{2}$ after three months of storage. After six months of storage, treatment effect towards shoot length was better for $\mathrm{V}_{1} \mathrm{~T}_{1}$, $\mathrm{V}_{1} \mathrm{~T}_{2}, \mathrm{~V}_{2} \mathrm{~T}_{1}, \mathrm{~V}_{2} \mathrm{~T}_{2}, \mathrm{~V}_{3} \mathrm{~T}_{1}, \mathrm{~V}_{3} \mathrm{~T}_{2}$ and $\mathrm{V}_{4} \mathrm{~T}_{1}$ than control. Indicating Azospirillum and PSB seed treatment has the ability for improving seedling shoot length. Similar higher values were also recorded for $V_{1} T_{1}, V_{3} T_{1}, V_{3} T_{2}$ than control (Table 3 ). The biofertilizer resulted in maximum reduction of seed rot and foot/root rot (Fusarium oxysporum) of bushbean stated by Khalequzaman and Hossain (2008).

The character root length was greatly improved by $T_{1}$ and $T_{2}$ for $V_{1}$ but for $V_{3} T_{1}$ and $V_{4} T_{2}$ the results were lower than control. Enhanced root length was also found after six months of storage. Higher values were recorded for $\mathrm{V}_{1} \mathrm{~T}_{1}, \mathrm{~V}_{1} \mathrm{~T}_{2}$ and $\mathrm{V}_{4} \mathrm{~T}_{1}$ than control (Table 4). Chandrasekhar (2003) observed that the plant growth parameters viz., root length and number of leaves per plant in green gram plants at 45 Days were significantly increased due to inoculation of $\mathrm{P}$ solubilizing fungal strains along with rock phosphate application as compared to rock phosphate alone (control).

Wheat varieties responded better towards seed treatment for the character fresh seedling weight after one month of storage for $V_{1} T_{1}, V_{1} T_{2}, V_{2} T_{1}, V_{2} T_{2}, V_{3} T_{1}$, $V_{3} T_{2}$ and $V_{4} T_{1}, V_{4} T_{2}$ than control (Table 5). After six months of storage, the seed treatment effect was observed for $\mathrm{V}_{1} \mathrm{~T}_{1}, \mathrm{~V}_{1} \mathrm{~T}_{2}, \mathrm{~V}_{3} \mathrm{~T}_{1}, \mathrm{~V}_{3} \mathrm{~T}_{2}$ and $\mathrm{V} 4 \mathrm{~T}_{1}, \mathrm{~V}_{4} \mathrm{~T}_{2}$ but slightly reduced in $\mathrm{V}_{2} \mathrm{~T}_{2}$. The variety-treatment interaction was greatly positive in $\mathrm{V}_{1} \mathrm{~T}_{1}, \mathrm{~V}_{1} \mathrm{~T}_{2}, \mathrm{~V}_{3} \mathrm{~T}_{1}, \mathrm{~V}_{3} \mathrm{~T}_{2}$ and $V_{4} T_{1}$ and $V_{4} T_{2}$ (Table 5). Mahfouz and Sharaf eldin (2007) have suggested the use of biofertilizers to increase the fresh weight of corn. The seedling dry weight, which is regarded as a great contributer towards seed vigour was also greatly enhanced by seed treatment. After one month of storage, $\mathrm{V}_{1} \mathrm{~T}_{1}, \mathrm{~V}_{1} \mathrm{~T}_{2}$ recorded such type of enhancement response of variety was for to be best both after three and six months of 
Table 1. Biofertilizers effect on speed of germination at after one, three and six months of storage (Three replicates).

\begin{tabular}{|c|c|c|c|c|c|c|c|c|c|c|c|c|}
\hline \multicolumn{13}{|c|}{ Speed of germination } \\
\hline \multirow{2}{*}{$\mathbf{V} / \mathbf{T}$} & \multicolumn{4}{|c|}{ After one month of storage } & \multicolumn{4}{|c|}{ After three months of storage } & \multicolumn{4}{|c|}{ After six months of storage } \\
\hline & T1 & T2 & T3 & Mean & T1 & T2 & T3 & Mean & T1 & $\mathbf{T 2}$ & T3 & Mean \\
\hline V1 & 2.26 & 2.33 & 2.20 & 2.26 & 2.20 & 2.06 & 2.07 & 2.11 & 2.08 & 1.93 & 1.82 & 1.94 \\
\hline $\mathrm{V} 2$ & 1.78 & 2.27 & 2.30 & 2.12 & 1.63 & 2.14 & 2.29 & 2.02 & 1.49 & 2.08 & 1.94 & 1.84 \\
\hline V3 & 2.07 & 2.23 & 1.75 & 2.02 & 1.80 & 2.29 & 1.84 & 1.97 & 1.45 & 1.90 & 1.63 & 1.66 \\
\hline V4 & 2.07 & 2.30 & 2.45 & 2.27 & 2.00 & 2.09 & 2.29 & 2.13 & 1.66 & 2.04 & 2.06 & 1.92 \\
\hline \multirow[t]{2}{*}{ Mean } & 2.04 & 2.28 & 2.18 & & 1.91 & 2.14 & 2.12 & & 1.67 & 1.99 & 1.86 & \\
\hline & $\mathbf{V}$ & $\mathbf{T}$ & VXT & & $\mathbf{V}$ & $\mathbf{T}$ & VXT & & $\mathbf{V}$ & $\mathbf{T}$ & VXT & \\
\hline $\operatorname{S.Em}( \pm)$ & 0.072 & 0.063 & 0.125 & & 0.028 & 0.024 & 0.049 & & 0.023 & 0.020 & 0.039 & \\
\hline $\mathrm{CD}(\mathrm{P}=0.05)$ & 0.211 & 0.183 & 0.366 & & 0.082 & 0.071 & 0.143 & & 0.066 & 0.058 & 0.115 & \\
\hline $\mathrm{CD}(\mathrm{P}=0.01)$ & 0.836 & 0.724 & 1.447 & & 0.326 & 0.283 & 0.565 & & 0.263 & 0.228 & 0.455 & \\
\hline
\end{tabular}

$\mathrm{V}_{1}$-Sonalika, $\mathrm{V}_{2}$ - PBW 443, $\mathrm{V}_{3}$, HD- 2821 and $\mathrm{V}_{4}-\mathrm{K} 9107$ and the treatments were- $\mathrm{T}_{1}$-Azospirillum, $\mathrm{T}_{2}-\mathrm{PSB}_{\text {and }} \mathrm{T}_{3}-\mathrm{Control}_{\text {. }}$

Table 2. Biofertilizers effect on germination percentage at after one, three and six months of storage (Three replicates).

\begin{tabular}{|c|c|c|c|c|c|c|c|c|c|c|c|c|}
\hline \multicolumn{13}{|c|}{ Germination \% } \\
\hline \multirow{2}{*}{$\mathbf{V} / \mathbf{T}$} & \multicolumn{4}{|c|}{ After one month of storage } & \multicolumn{4}{|c|}{ After three months of storage } & \multicolumn{4}{|c|}{ After six months of storage } \\
\hline & T1 & T2 & T3 & Mean & T1 & T2 & T3 & Mean & T1 & $\mathbf{T 2}$ & T3 & Mean \\
\hline V1 & 96.67 & 96.67 & 86.67 & 93.33 & 83.33 & 83.33 & 76.67 & 81.11 & 76.67 & 73.33 & 70.00 & 73.33 \\
\hline $\mathrm{V} 2$ & 96.67 & 86.67 & 96.67 & 93.33 & 83.33 & 73.33 & 83.33 & 80.00 & 76.67 & 56.67 & 73.33 & 68.89 \\
\hline $\mathrm{V} 3$ & 96.67 & 93.33 & 93.33 & 94.44 & 83.33 & 83.33 & 76.67 & 81.11 & 73.33 & 73.33 & 63.33 & 70.00 \\
\hline V4 & 80.00 & 83.33 & 90.00 & 84.44 & 70.00 & 70.00 & 76.67 & 72.22 & 56.67 & 56.67 & 63.33 & 58.89 \\
\hline \multirow[t]{2}{*}{ Mean } & 92.50 & 90.00 & 91.67 & & 80.00 & 77.50 & 78.33 & & 70.83 & 65.00 & 67.50 & \\
\hline & $\mathrm{V}$ & $\mathrm{T}$ & VXT & & $\mathrm{V}$ & $\mathrm{T}$ & VXT & & $\mathrm{V}$ & $\mathrm{T}$ & VXT & \\
\hline S.Em $( \pm)$ & 2.222 & 1.925 & 3.849 & & 2.003 & 1.735 & 3.469 & & 2.079 & 1.800 & 3.600 & \\
\hline $\mathrm{CD}(\mathrm{P}=0.05)$ & 6.486 & 5.617 & 11.234 & & 5.847 & 5.063 & 10.127 & & 6.067 & 5.254 & 10.509 & \\
\hline $\mathrm{CD}(\mathrm{P}=0.01)$ & 25.656 & 22.219 & 44.437 & & 23.126 & 20.028 & 40.055 & & 1.468 & 1.271 & 2.542 & \\
\hline
\end{tabular}

$\mathrm{V}_{1}$-Sonalika, $\mathrm{V}_{2}$ - PBW 443, $\mathrm{V}_{3}$, HD- 2821 and $\mathrm{V}_{4}$ - K 9107 and the treatments were- $\mathrm{T}_{1}$-Azospirillum, $\mathrm{T}_{2}$ - PSB and $\mathrm{T}_{3}$-Control.

Table 3. Biofertilizers effect on shoot length $(\mathrm{cm})$ at after one, three and six months of storage (Three replicates).

\begin{tabular}{|c|c|c|c|c|c|c|c|c|c|c|c|c|}
\hline \multicolumn{13}{|c|}{ Shoot length $(\mathrm{cm})$} \\
\hline \multirow{2}{*}{$\mathbf{V} / \mathbf{T}$} & \multicolumn{4}{|c|}{ After one month of storage } & \multicolumn{4}{|c|}{ After three months of storage } & \multicolumn{4}{|c|}{ After six months of storage } \\
\hline & T1 & $\mathbf{T 2}$ & T3 & Mean & $\mathbf{T 1}$ & $\mathbf{T 2}$ & T3 & Mean & T1 & $\mathbf{T 2}$ & T3 & Mean \\
\hline V1 & 15.47 & 11.37 & 10.17 & 12.34 & 14.43 & 10.47 & 9.53 & 11.48 & 10.62 & 5.89 & 6.00 & 7.50 \\
\hline $\mathrm{V} 2$ & 15.67 & 14.99 & 12.27 & 14.31 & 12.93 & 12.50 & 11.73 & 12.39 & 8.72 & 8.20 & 7.22 & 8.05 \\
\hline $\mathrm{V} 3$ & 15.07 & 16.07 & 10.30 & 13.81 & 13.67 & 13.83 & 10.43 & 12.64 & 9.45 & 9.85 & 5.99 & 8.43 \\
\hline V4 & 14.00 & 9.34 & 9.09 & 10.81 & 12.30 & 8.90 & 9.00 & 10.07 & 8.19 & 5.37 & 5.33 & 6.30 \\
\hline \multirow[t]{2}{*}{ Mean } & 15.05 & 12.94 & 10.46 & & 13.33 & 11.43 & 10.18 & & 9.25 & 7.33 & 6.14 & \\
\hline & $\mathrm{V}$ & $\mathrm{T}$ & VXT & & V & $\mathrm{T}$ & VXT & & V & $\mathrm{T}$ & VXT & \\
\hline S.Em( $( \pm)$ & 0.282 & 0.245 & 0.489 & & 0.125 & 0.109 & 0.217 & & 0.127 & 0.110 & 0.220 & \\
\hline $\mathrm{CD}(\mathrm{P}=0.05)$ & 0.825 & 0.714 & 1.428 & & 0.366 & 0.317 & 0.634 & & 0.371 & 0.321 & 0.643 & \\
\hline $\mathrm{CD}(\mathrm{P}=0.01)$ & 3.261 & 2.824 & 5.649 & & 1.447 & 1.253 & 2.506 & & 1.468 & 1.271 & 2.542 & \\
\hline
\end{tabular}

$\mathrm{V}_{1}$-Sonalika, $\mathrm{V}_{2}$ - PBW 443, $\mathrm{V}_{3}$, HD- 2821 and $\mathrm{V}_{4}-\mathrm{K} 9107$ and the treatments were- $\mathrm{T}_{1}$-Azospirillum, $\mathrm{T}_{2}-\mathrm{PSB}_{\text {and }} \mathrm{T}_{3}-\mathrm{Control}$

Table 4. Biofertilizers effect on root length $(\mathrm{cm})$ at after one, three and six months of storage (Three replicates).

\begin{tabular}{|c|c|c|c|c|c|c|c|c|c|c|c|c|}
\hline \multirow{3}{*}{$\mathbf{V} / \mathbf{T}$} & \multicolumn{8}{|c|}{ Root length $(\mathrm{cm})$} & \multirow{2}{*}{\multicolumn{4}{|c|}{ After six months of storage }} \\
\hline & \multicolumn{4}{|c|}{ After one month of storage } & \multicolumn{4}{|c|}{ After three months of storage } & & & & \\
\hline & T1 & $\mathbf{T 2}$ & T3 & Mean & T1 & $\mathbf{T 2}$ & T3 & Mean & T1 & $\mathbf{T 2}$ & T3 & Mean \\
\hline V1 & 13.36 & 13.43 & 11.38 & 12.72 & 11.93 & 12.53 & 10.83 & 11.77 & 10.87 & 11.47 & 9.00 & 10.44 \\
\hline $\mathrm{V} 2$ & 15.03 & 13.83 & 14.87 & 14.58 & 12.80 & 12.80 & 13.33 & 12.98 & 11.67 & 11.80 & 12.03 & 11.83 \\
\hline V3 & 12.42 & 13.47 & 13.00 & 12.96 & 12.20 & 12.93 & 12.27 & 12.47 & 10.30 & 10.33 & 10.67 & 10.43 \\
\hline V4 & 15.40 & 10.17 & 11.09 & 12.22 & 13.80 & 9.93 & 9.97 & 11.23 & 12.60 & 8.30 & 8.33 & 9.74 \\
\hline \multirow{2}{*}{ Mean } & 14.05 & 12.73 & 12.58 & & 12.68 & 12.05 & 11.60 & & 11.36 & 10.48 & 10.01 & \\
\hline & $\mathrm{V}$ & $\mathrm{T}$ & VXT & & V & $\mathrm{T}$ & VXT & & V & $\mathrm{T}$ & VXT & \\
\hline S.Em( $( \pm)$ & 0.207 & 0.179 & 0.358 & & 0.165 & 0.143 & 0.286 & & 0.124 & 0.107 & 0.214 & \\
\hline $\mathrm{CD}(\mathrm{P}=0.05)$ & 0.604 & 0.523 & 1.045 & & 0.482 & 0.418 & 0.835 & & 0.361 & 0.313 & 0.626 & \\
\hline $\mathrm{CD}(\mathrm{P}=0.01)$ & 2.387 & 2.068 & 4.135 & & 1.907 & 1.652 & 3.303 & & 1.428 & 1.237 & 2.474 & \\
\hline
\end{tabular}

$\mathrm{V}_{1}$-Sonalika, $\mathrm{V}_{2}$ - PBW 443, $\mathrm{V}_{3}$, HD- 2821 and $\mathrm{V}_{4}-\mathrm{K} 9107$ and the treatments were- $\mathrm{T}_{1}$-Azospirillum, $\mathrm{T}_{2}$ - PSB and $\mathrm{T}_{3}$-Control.

storage (Table 6). The similar work was done in case of corn and showed the biofertilizers increased the dry weight proposed by Mahfouz and Sharaf eldin (2007). The study revealed that maximum germination (92\%), viability $(95 \%)$, germination speed (1.58), germination energy $(70.0 \%)$ were recorded in polybags under complete darkness in Aconitum heterophyllum wall ex. Royle while minimum were recorded under partial light in cloth bags. Similarly, in case of Podophyllum hexandrum same results were obtained stated by 
Table 5. Biofertilizers effect on fresh weight $(\mathrm{g})$ at after one, three and six months of storage (Three replicates).

\begin{tabular}{|c|c|c|c|c|c|c|c|c|c|c|c|c|}
\hline \multicolumn{13}{|c|}{ Fresh weight (g) } \\
\hline \multirow{2}{*}{$\mathbf{V} / \mathbf{T}$} & \multicolumn{4}{|c|}{ After one month of storage } & \multicolumn{4}{|c|}{ After three months of storage } & \multicolumn{4}{|c|}{ After six months of storage } \\
\hline & $\mathbf{T 1}$ & $\mathbf{T 2}$ & T3 & Mean & $\mathbf{T 1}$ & $\mathbf{T} 2$ & T3 & Mean & T1 & $\mathbf{T} 2$ & T3 & Mean \\
\hline V1 & 1.21 & 1.30 & 0.86 & 1.12 & 1.07 & 1.17 & 0.79 & 1.01 & 1.06 & 1.14 & 0.83 & 1.01 \\
\hline $\mathrm{V} 2$ & 0.74 & 0.73 & 0.71 & 0.73 & 0.69 & 0.66 & 0.67 & 0.67 & 0.67 & 0.65 & 0.67 & 0.66 \\
\hline $\mathrm{V} 3$ & 1.15 & 1.13 & 0.88 & 1.05 & 1.17 & 1.17 & 0.82 & 1.05 & 1.10 & 1.07 & 0.85 & 1.01 \\
\hline V4 & 0.93 & 0.90 & 0.80 & 0.88 & 0.92 & 0.85 & 0.76 & 0.85 & 0.85 & 0.83 & 0.74 & 0.81 \\
\hline \multirow[t]{2}{*}{ Mean } & 1.01 & 1.01 & 0.81 & & 0.96 & 0.96 & 0.76 & & 0.92 & 0.92 & 0.77 & \\
\hline & V & $\mathrm{T}$ & VXT & & V & $\mathrm{T}$ & VXT & & V & $\mathrm{T}$ & VXT & \\
\hline $\operatorname{S.Em}( \pm)$ & 0.027 & 0.024 & 0.047 & & 0.015 & 0.013 & 0.027 & & 0.021 & 0.018 & 0.036 & \\
\hline $\mathrm{CD}(\mathrm{P}=0.05)$ & 0.080 & 0.069 & 0.138 & & 0.045 & 0.039 & 0.078 & & 0.060 & 0.052 & 0.104 & \\
\hline $\mathrm{CD}(\mathrm{P}=0.01)$ & 0.316 & 0.273 & 0.547 & & 0.178 & 0.154 & 0.309 & & 0.238 & 0.206 & 0.411 & \\
\hline
\end{tabular}

$\mathrm{V}_{1}$-Sonalika, $\mathrm{V}_{2}$ - PBW 443, $\mathrm{V}_{3}$, HD- 2821 and $\mathrm{V}_{4}-\mathrm{K} 9107$ and the treatments were- $\mathrm{T}_{1}$-Azospirillum, $\mathrm{T}_{2}$ - PSB and $\mathrm{T}_{3}$-Control.

Table 6. Biofertilizers effect on dry weight (g) at after one, three and six months of storage (Three replicates).

\begin{tabular}{|c|c|c|c|c|c|c|c|c|c|c|c|c|}
\hline \multicolumn{13}{|c|}{ Dry weight (g) } \\
\hline \multirow{2}{*}{$\mathbf{V} / \mathbf{T}$} & \multicolumn{4}{|c|}{ After one month of storage } & \multicolumn{4}{|c|}{ After three months of storage } & \multicolumn{4}{|c|}{ After six months of storage } \\
\hline & T1 & $\mathbf{T} 2$ & T3 & Mean & T1 & $\mathbf{T} 2$ & T3 & Mean & T1 & T2 & T3 & Mean \\
\hline V1 & 0.18 & 0.19 & 0.13 & 0.17 & 0.17 & 0.17 & 0.10 & 0.15 & 0.14 & 0.15 & 0.07 & 0.12 \\
\hline $\mathrm{V} 2$ & 0.12 & 0.13 & 0.41 & 0.22 & 0.11 & 0.10 & 0.10 & 0.10 & 0.06 & 0.06 & 0.04 & 0.05 \\
\hline V3 & 0.17 & 0.13 & 0.13 & 0.14 & 0.17 & 0.12 & 0.12 & 0.14 & 0.13 & 0.09 & 0.07 & 0.09 \\
\hline V4 & 0.13 & 0.11 & 0.13 & 0.12 & 0.12 & 0.10 & 0.13 & 0.12 & 0.06 & 0.05 & 0.07 & 0.06 \\
\hline \multirow{2}{*}{ Mean } & 0.15 & 0.14 & 0.20 & & 0.14 & 0.12 & 0.11 & & 0.09 & 0.08 & 0.06 & \\
\hline & $\mathrm{V}$ & $\mathrm{T}$ & VXT & & $\mathrm{V}$ & $\mathrm{T}$ & VXT & & V & $\mathrm{T}$ & VXT & \\
\hline $\operatorname{S.Em}( \pm)$ & 0.049 & 0.042 & 0.084 & & 0.002 & 0.002 & 0.003 & & 0.009 & 0.008 & 0.016 & \\
\hline $\mathrm{CD}(\mathrm{P}=0.05)$ & 0.142 & 0.123 & 0.247 & & 0.005 & 0.004 & 0.009 & & 0.027 & 0.024 & 0.047 & \\
\hline $\mathrm{CD}(\mathrm{P}=0.01)$ & 0.563 & 0.488 & 0.975 & & 0.020 & 0.017 & 0.035 & & 0.108 & 0.094 & 0.187 & \\
\hline
\end{tabular}

$\mathrm{V}_{1}$-Sonalika, $\mathrm{V}_{2}-\mathrm{PBW} 443, \mathrm{~V}_{3}$, HD- 2821 and $\mathrm{V}_{4}-\mathrm{K} 9107$ and the treatments were- $\mathrm{T}_{1}$-Azospirillum, $\mathrm{T}_{2}$ - PSB and $\mathrm{T}_{3}$ - Control.

Table 7. Biofertilizers effect on Vigour index at after one, three and six months of storage.

\begin{tabular}{|c|c|c|c|c|c|c|c|c|c|c|c|c|}
\hline \multicolumn{13}{|c|}{ Vigour index } \\
\hline \multirow{2}{*}{$\mathrm{V} / \mathrm{T}$} & \multicolumn{3}{|c|}{ After one month of storage } & \multirow[b]{2}{*}{ Mean } & \multicolumn{4}{|c|}{ After three months of storage } & \multicolumn{4}{|c|}{ After six months of storage } \\
\hline & T1 & T2 & T3 & & T1 & T2 & T3 & Mean & T1 & T2 & T3 & Mean \\
\hline V1 & 2785 & 2402 & 1868 & 2352 & 2198 & 1915 & 1562 & 1892 & 1648 & 1270 & 1049 & 1322 \\
\hline $\mathrm{V} 2$ & 2965 & 2498 & 2624 & 2696 & 2146 & 1856 & 2088 & 2030 & 1563 & 1132 & 1409 & 1368 \\
\hline V3 & 2660 & 2757 & 2172 & 2530 & 2154 & 2230 & 1739 & 2041 & 1449 & 1482 & 1053 & 1328 \\
\hline V4 & 2347 & 1631 & 1812 & 1930 & 1821 & 1318 & 1454 & 1531 & 1179 & 774 & 865 & 939 \\
\hline \multirow[t]{2}{*}{ Mean } & 2689 & 2322 & 2119 & & 2080 & 1830 & 1711 & & 1459 & 1165 & 1094 & \\
\hline & V & $\mathrm{T}$ & VXT & & $\mathrm{V}$ & $\mathrm{T}$ & VXT & & $\mathrm{V}$ & $\mathrm{T}$ & VXT & \\
\hline S.Em $( \pm)$ & 64.507 & 55.865 & 111.730 & & 46.73 & 40.47 & 80.94 & & 36.57 & 31.67 & 63.35 & \\
\hline $\mathrm{CD}(\mathrm{P}=0.05)$ & 188.28 & 163.06 & 326.12 & & 136.4 & 118.1 & 236.2 & & 106.8 & 92.45 & 184.9 & \\
\hline $\mathrm{CD}(\mathrm{P}=0.01)$ & 744.75 & 644.97 & 1289.94 & & 539.5 & 467.2 & 934.4 & & 422.3 & 365.7 & 731.4 & \\
\hline
\end{tabular}

$\mathrm{V}_{1}$-Sonalika, $\mathrm{V}_{2}$ - PBW 443, $\mathrm{V}_{3}$, HD- 2821 and $\mathrm{V}_{4}-\mathrm{K} 9107$ and the treatments were- $\mathrm{T}_{1}$-Azospirillum, $\mathrm{T}_{2}$ - PSB and $\mathrm{T}_{3}$ - Control.

Verma et al. (2011). A storage experiment was conducted to understand the effect of organic and integrated management practices of seed production and storage containers along with organic (insecticide and fungicide) and organic (botanicals) as seed treatments on seed viability of scented rice cv. Mugad sugandha for 20 months reported by Raikar et al. (2011). Chattha et al. (2012) observed the effect of different packing materials (metal bin, earthen bin, plastic bag, cloth bag and gunny bag) and grain moisture content at packing $(10 \%$ and $16 \%)$ on viability of wheat seed for ten months of storage.

The seedling quality parameter, vigour index, where two important seed quality parameters like germination percentage and seedling length was taken into account to find out the response of varieties under study towards maintaining or enhancing vigour status after six months of storage. It is noted that, vigour index was greatly enhanced in case of $V_{1} T_{2}, V_{1} T_{2}, V_{3} T_{1}, V_{3} T_{2}$ after one, three and six months of storage. After six months of storage, in comparison to control, all the treatments recorded maximum value except $\mathrm{V}_{4} \mathrm{~T}_{2}$ and $\mathrm{V}_{2} \mathrm{~T}_{2}$. Other $\mathrm{V}_{1} \mathrm{~T}_{1}, \mathrm{~V}_{1} \mathrm{~T}_{2}, \mathrm{~V}_{2} \mathrm{~T}_{1}, \mathrm{~V}_{3} \mathrm{~T}_{1}, \mathrm{~V}_{3} \mathrm{~T}_{2}$ and $\mathrm{V}_{4} \mathrm{~T}_{1}$ all recorded better value than control. Similar results were reported by Albrecht et al. (1981), Mishra et al. (1998) and Rout et al. (2001) in case of maize. The highest germination, normal seedlings and vigour index which were followed by polythene bag, where Gunny bag showed the lowest germination, normal seedlings and vigour index upto 60 days after storage by Khalequzzaman et al. (2012).

\section{Conclusion}

From findings it was revealed that, Azospirillum and PSB seed inoculation can be recommended for wheat for better seedling growth storability of seed. It should also be noted that, these bio-fertilizers are less expensive than inorganic manures and they are also ecofriendly. More number of wheat genotypes may be used in future for recommendation of actual bio- 
fertilizers dose for yield enhancement.

\section{REFERENCES}

Albrecht, S.L., Okon, Lonnquist L and Burries, R.H. (1981). Nitrogen fixation by corn Azospirillum associations in a temperature climate. Crop Science, 21: 301-306.

Chandrasekhar, B.S. (2003). Studies on mineral phosphate solubilizing fungi from vertisols of Northern Karnataka and other Biofertilzier potential. Ph.D. Thesis, Univ. Agric. Sci., Dharwad, Karnataka, India.

Chattha, S.H., Jamali, L.A., Ibupoto, K.A. and Mangio, H.R. (2012). Effect of different packing materials and Storage conditions on the viability of wheat Seed (TD-1 variety). Sci. Tech. and Dev., 31 (1): 10-18.

Cochran, W.G and Cox, G.M. (1963). Experimental Designs. In: $1^{\text {st }}$ India Ed. Asia publishing House, Bombay, Calcutta, New Delhi and Madras.

Garcia, de Salamone, I.E. Hynes, R.K. and Nelson, L. M. (2001). Cytokinins production by plant growth promoting rhizobacteria and selected mutants. Can. J. Microbiol., 47: 404-411.

Gomez, K.A. and Gomez, A.A. (1984). Statistical Procedures for Agricultural Research, $2^{\text {nd }}$ edn. Singapore: John Wiley \& Sons.

Javed, S. and Panwar, A. (2013). Effect of biofertilizer, vermicompost and chemical fertilizer on different biochemical parameters of Glycine max and Vigna mungo. Recent Research in Science and Technology. 5 (1): 4044.

K.M. Khalequzaman and I. Hossain (2008). Effect of Seed Treatment with Rhizobium Strains and Biofertilizers on foot/root rot and Yield of Bushbean in Fusarium oxysporum infested soil. J. Agric. Res., 46 (1): 55-65

Khalequzzaman, K.M., Rashid, M.M., Hasan, M.A. and Reza, M. M. A. (2012). Effect of storage containers and storage periods on the seed quality of french bean (Phaseolus vulgaris). Bangladesh J. Agril. Res., 37 (2): 195-205.

Mahfouz S.A. and Sharaf-Eldin M.A. (2007). Effect of mineral vs. biofertilizer on growth, yield and essential oil content of fennel (Foeniculum vulgare Mill.). Int Agrophys. 21: $361-366$.
Mishra, M.; Patyonshi, A.K. and Jena, D. (1998). Effect of biofertilizers on productivity of maizeb (zea mays), Indian Journal of Agronomy. 43: 307-310.

Narula, N. Kumar, V. Singh, B. Bhatia, R. and Lakshminarayana, K. (2005). Impact of Biofertilizer on rain yield in spring wheat under varying fertility condition and wheat cotton rotation. Archiv. Agron. and Soil Sci., 51: 79-89.

Nautiyal, C.S. Bhadauria, S. Kumar, P.L. Momdal, R. and Verma, D. (2000). Stress induced phosphate solubilization in bacteria isolated from alkaline soils. FEMS Micro-biology Lett.182-:291-296.

Pauw, De. Mirghasemi, E.A. Ghaffari, A. and Nseir, B. (2008). Agro ecological zones of Karkheh River, Basin. Research Report, ICARDA, Aleppo, Syria.

Raikar, S.D., Vyakarnahal, B.S., Biradar, D. P., Deshpande, V.K. and Janagoudar, B.S. (2011). Effect of seed source, containers and seed treatment with chemical and biopesticide on storability of scented rice $\mathrm{Cv}$. Mugad sugandha. Karnataka J. Agric. Sci., 24 (4): 448-454.

Rout, D., Satalathy, M.R.; and Mohapatra, B.K. (2001). Effect of biofertilizers on nitrogen economy in maize. Madras Agricultural Journal. 88:7-9.

Snedecor, G and Cochran, W.G. 1967. Statistical methods, Oxford and IBH Publishing Company, Bombay, pp. 135 $-197$.

Strelec, I., Popovvic, R., Ivanisic, I., Jurkovic, V., Jurkovic, Z., Hardi, Z.U. and Sabo,M. (2010). Influence of temperature and relative humidity on grain moisture, germination and vigour of three wheat cultivars during one year storage. Poljoprivreda, 16 (2): 20-24.

Subba Rao, N.S. (1993). Biofertilizers in Agriculture and Forestry, Oxford and IBM publishing co. (p) Ltd. 3 rd edition.

Verma, N.K., Verma, A.K. and Kumar, D. (2011). Effect of storage containers on seed germination and viability in Aconitum heterophyllum and Podophyllum hexandrum - endangered medicinal plants species of Himalayan region. Biological Forum - An International Journal, 1 (1): 8-11.

Vessey, J.K. (2003). Plant growth promoting rhizobacteria as biofertilizer. Plant and Soil. 255: 571-586. 